\title{
Laser Ablation split stream for in situ sulfur isotope and elemental analysis
}

\author{
MARCUS OELZE ${ }^{1}$, DANIEL A. FRICK ${ }^{1}$ AND SARAH A. \\ GLEESON $^{1,2}$ \\ ${ }^{1}$ GFZ German Research Centre for Geosciences \\ ${ }^{2}$ Freie Universität Berlin, Germany \\ Presenting Author: oelze@gfz-potsdam.de
}

Studies of sulfur isotopes provide important insights into a variety of geological, geochemical and biological systems. The shift in the $\mathrm{S}$ isotopic composition is mainly induced by oxidation or reduction reactions. Sulfur isotopes can trace these redox reactions from high temperature magmatic systems to low temperature sedimentary diagenesis as well as reactions in hydrothermal systems at intermediate temperatures. Trace element chemistry of sulfur minerals provide an additional valuable tool to constrain sulfide mineral origin and formation history. Here, we combine these two tools using laser ablation split stream to measure simultaneously the $\mathrm{S}$ isotopic composition and the corresponding trace element chemistry. This technique enables us to derive more information from single measurements and further detect changes in the composition of the ablated material and reduce the measurement uncertainty. In situ sulfur (S) isotope ratios and trace element chemistry were simultaneously determined on a wide selection of different (natural) sulfides and sulfates using femtosecond laser ablation split stream (fsLASS) inductively coupled plasma mass spectrometry (ICP-MS). The laser aerosol is split between a sequential quadrupole ICP-MS for trace element quantification and a multi-collector ICP-MS (MC-ICP-MS) for stable sulfur isotope ratio measurements. This LASS method is able to simultaneously determine $\mathrm{S}$ isotope ratios and element chemistry without a compromise on the measurement precision and measurement accuracy of the $\mathrm{S}$ isotope ratios. LASS shows overall lower sensitivity and higher limits of detection in comparison to direct trace element determinations using LA-ICPMS only, due to the lower amounts of sample introduced into the ICP-qMS. This compromise is acceptable given the additional information gained from obtaining simultaneously both the isotopic and elemental compositions. 